\title{
Tumor suppressor FLCN inhibits tumorigenesis of a FLCN-null renal cancer cell line and regulates expression of key molecules in TGF- $\beta$ signaling
}

\author{
Seung-Beom Hong1', HyoungBin Oh', Vladimir A Valera', Jaime Stull', Duy-Tan Ngo', Masaya Baba', Maria J Merino², \\ W Marston Linehan ${ }^{1}$ and Laura S Schmidt*1,3
}

\begin{abstract}
Background: Germline mutations in the FLCN gene are responsible for the development of fibrofolliculomas, lung cysts and renal neoplasia in Birt-Hogg-Dube' (BHD) syndrome. The encoded protein folliculin (FLCN) is conserved across species but contains no classic motifs or domains and its function remains unknown. Somatic mutations or loss of heterozygosity in the remaining wild type copy of the FLCN gene have been found in renal tumors from BHD patients suggesting that FLCN is a classic tumor suppressor gene.

Results: To examine the tumor suppressor function of FLCN, wild-type or mutant FLCN (H255R) was stably expressed in a FLCN-null renal tumor cell line, UOK257, derived from a BHD patient. When these cells were injected into nude mice, tumor development was inversely dependent upon the level of wild-type FLCN expression. We identified genes that were differentially expressed in the cell lines with or without wild-type FLCN, many of which are involved in TGF- $\beta$ signaling, including TGF- $\beta 2$ (TGFB2), inhibin $\beta$ A chain (INHBA), thrombospondin 1 (THBS1), gremlin (GREM1), and SMAD3. In support of the in vitro data, TGFB2, INHBA, THBS1 and SMAD3 expression levels were significantly lower in BHDassociated renal tumors compared with normal kidney tissue. Although receptor mediated SMAD phosphorylation was not affected, basal and maximal TGF- $\beta$-induced levels of TGFB2, INHBA and SMAD7 were dramatically reduced in FLCN-null cells compared with FLCN-restored cells. Secreted TGF- $\beta 2$ and activin A (homo-dimer of INHBA) protein levels were also lower in FLCN-null cells compared with FLCN-restored cells. Consistent with a growth suppressive function, activin A (but not TGF- $\beta 2$ ) completely suppressed anchorage-independent growth of FLCN-null UOK257 cells.
\end{abstract}

Conclusions: Our data demonstrate a role for FLCN in the regulation of key molecules in TGF- $\beta$ signaling and confirm deregulation of their expression in BHD-associated renal tumors. Thus, deregulation of genes involved in TGF- $\beta$ signaling by FLCN inactivation is likely to be an important step for tumorigenesis in BHD syndrome.

\section{Background}

Birt-Hogg-Dubé (BHD) syndrome is a familial disorder that predisposes patients to develop hair follicle hamartomas (84-90\% penetrance), lung cysts (85\% penetrance) and renal neoplasia (29-34\% penetrance) [1-5]. BHD patients are at risk to develop bilateral, multifocal renal tumors with a variety of histologies, mainly chromophobe (34\%) and oncocytic hybrid (50\%) tumors with features of both chromophobe renal cell carcinoma (RCC)

\footnotetext{
* Correspondence: schmidtl@mail.nih.gov

1 Urologic Oncology Branch, Center for Cancer Research, National Cancer Institute, 10 Center Drive MSC1107, 10/CRC/1W-5940, Bethesda, MD 20892 USA

Full list of author information is available at the end of the article
}

and renal oncocytoma. Clear cell and papillary RCC as well as renal oncocytomas are also found in BHD patients at a low frequency [6]. The BHD syndrome locus was mapped to chromosome $17 \mathrm{p} 11.2$ by linkage analysis in BHD families, and germline mutations in a novel gene FLCN (alias BHD), were identified and characterized [5,711]. Most BHD families carry germline mutations predicted to truncate the encoded protein, folliculin (FLCN), including insertion/deletion, nonsense, and splice-site mutations reported in several large BHD cohorts $[4,5,11]$. Either somatic "second hit" mutations predicted to truncate the protein or loss of heterozygosity at the BHD syndrome locus was identified in $70 \%$ of renal tumors from 
BHD patients [12] supporting a tumor suppressor function for FLCN.

Two naturally-occurring animal models have been described that show phenotypes similar to BHD patients. The Nihon rat model develops renal carcinoma with clear cell histology by 6 months of age and harbors a cytosine insertion mutation in exon 3 of rat Flcn [13]. A canine model of BHD, which develops renal cystadenocarcinoma and nodular dermatofibrosis (RCND), carries a germline missense mutation (H255R) in canine Flcn [14]. Recently, we and others described a conditional Flcn knockout mouse model in which Flcn inactivation was targeted to mouse kidney using the Cre-lox site-specific recombination system. The affected mice displayed renal hyperplasia, formation of multiple cysts and renal dysfunction, suggesting important roles for Flcn in regulation of renal cell proliferation $[15,16]$. No tumors formed before the animals died at 3 weeks of age due to renal failure, and therefore the mechanism by which Flcn inactivation leads to kidney cancer could not be examined in this in vivo model. However, recently we and others have reported that mice heterozygous for Flcn develop renal cysts and tumors as they age beyond a year [17-19], with demonstrated loss of the wild type copy of Flcn (17). These Flcn +/- mouse models more closely mimic BHD syndrome in the human, albeit with a long latency.

FLCN encodes a $64 \mathrm{kDa}$ protein with no characteristic functional domains, which forms a complex with novel folliculin-interacting proteins 1 and 2 (FNIP1 and FNIP2), and 5'-AMP-activated protein kinase (AMPK), an important energy sensor in cells that negatively regulates mammalian target of rapamycin (mTOR) [20,21]. Phosphorylation of FLCN and FNIP1 was regulated by AMPK and mTOR activities suggesting a functional relationship with the AMPK-mTOR pathway. Interestingly, activation of mTOR downstream signaling molecules was seen in kidney-targeted $B H D$ conditional knockout mouse kidneys $[15,16]$. In addition, the renal tumors from BHD patients showed increased phosphorylation of mTOR [15]. In contrast to these results, it was suggested that yeast homologs of FLCN and TSC1/2 may have opposing roles in amino acid homeostasis [22]. The cysts and renal tumors derived from the Flcn heterozygous mice described by Hartman et al. showed reduced phospho-S6R suggesting diminished mTOR activation [18]. On the other hand Hasumi and coworkers found upregulation of both mTORC1 and mTORC2 pathways in kidney tumors from $\mathrm{FlCn}^{d /+}$ mice [17]. Hudon et al. suggest that up or down regulation of mTOR by inactivation of Flcn in a mouse model may be context-dependent [19]. Thus it is possible that mTOR signaling is regulated differently by FLCN depending on cell types or experimental conditions.
A renal cancer cell line (UOK257) established from a BHD patient was recently developed and characterized [23]. UOK257 cells harbor a cytosine insertion in a (poly)C tract, the frequently mutated "hot spot" within exon 11 of FLCN (c.1285dupC), and have lost the wildtype copy of FLCN. Cytogenetic analysis revealed that the cell line was nearly triploid displaying multiple unbalanced translocations and deletions of chromosomes. The MYC copy number was heterogeneous in UOK257 cells ranging from 3 to 5 copies. These cells formed tumors in immunodeficient mice (SCID/BEIG) exhibiting predominantly atypical clear epithelial cell type histology, as well as a variety of other histologic types including tubular papillary, and foci reminiscent of chromophobe RCC, all of which resemble the histologies within the tumor from which the cell line was derived [23].

In the current study, in order to investigate the tumor suppressor function of FLCN we have introduced wildtype FLCN into UOK257 cells and compared their growth in vitro and in vivo. We found that wild-type FLCN suppressed tumor cell growth in vivo, confirming the tumor suppressor function of FLCN. In addition, we employed gene expression microarray analysis to identify novel downstream target genes of FLCN. Among the differentially expressed genes, we identified several critical genes involved in TGF- $\beta$ signaling including TGFB2, INHBA, THBS1, GREM1 and SMAD3. Since deregulation of TGF- $\beta$ signaling is important in tumorigenesis and tumor progression, we characterized the expression of these genes in FLCN-null and FLCN-expressing cultured cells as well as in renal tumors surgically removed from BHD patients. In addition, we examined the growth suppressive effect of activin A in the FLCN-null cell line and investigated receptor mediated TGF- $\beta$ signaling in FLCN-null and FLCN-restored cell lines.

\section{Results}

Wild-type FLCN expression was restored in UOK257 cells by lentiviral vectors

To evaluate the tumor suppressor function of FLCN, wild-type or mutant (H255R) FLCN cDNA was introduced into the FLCN-null UOK257 cells using lentiviral vectors. Four clones expressing wild-type FLCN (UOK257-2, -3, -4, and -6) and one clone expressing mutant FLCN-H255R (UOK257-H255R; Flcn missense mutation responsible for canine RCND) were isolated and compared to the parental UOK257 cells (UOK257-P). FLCN protein expression was measured by Western blot analysis using a mouse monoclonal anti-FLCN antibody. Relatively high levels of FLCN protein were detected in the UOK257-2, -4 and -6 cells but very low levels of FLCN protein were detected in the UOK257-3 and UOK257-H255R cells (Fig. 1A). FLCN mRNA expression 

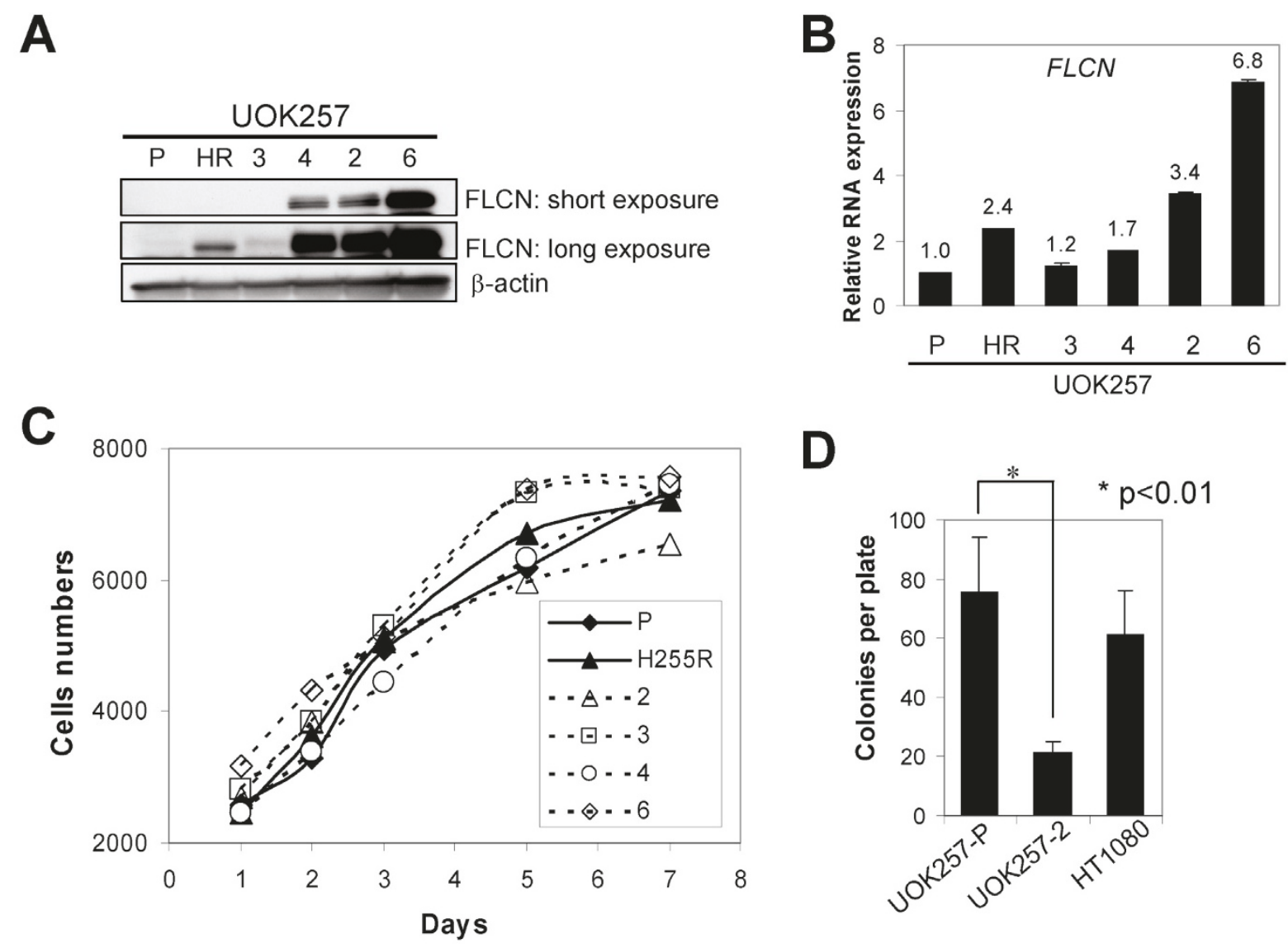

D

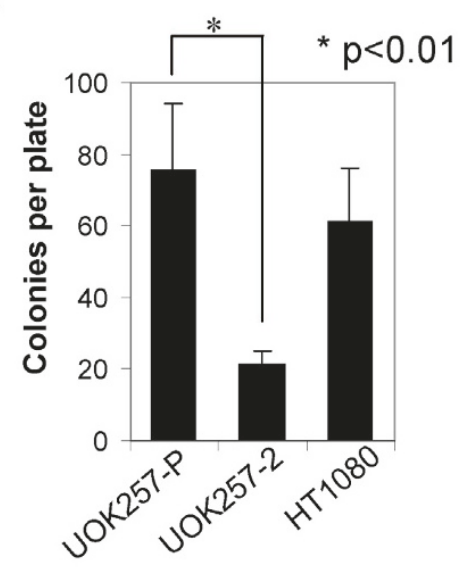

Figure 1 Characterization of the UOK257 cell lines. (A) FLCN protein expression in the UOK257 cell lines restored with wildtype or mutant FLCN. P, parental; HR, FLCN (H255R) mutant; 2, 3, 4 and 6, wildtype FLCN. (B) FLCN mRNA level was measured by quantitative RT-PCR. Columns, mean; bars, +SD $(n=3)$. (C) Cell growth of the UOK257 cell lines in vitro. Points, mean $(n=4)$. (D) Colony formation assay of the UOK257 cell lines and control HT1080 cell line in soft-agar culture. Columns, mean; bars, $+S D(n=3)$.

from both the transgene and endogenous $F L C N$ was measured by quantitative RT-PCR. The total FLCN mRNA expression was increased by the expression of the wildtype $F L C N$ or mutant $F L C N-H 255 R$ transgene to varying degrees in the cell lines $(\mathrm{P}=3<4<\mathrm{H} 255 \mathrm{R}<2<6$ ) (Fig. 1B).

\section{Anchorage independent but not dependent growth of UOK257 cells was inversely correlated with wild-type FLCN expression}

We examined whether introduction of wild-type or mutant FLCN could affect anchorage dependent and independent growth of UOK257 cells. Anchorage dependent growth of UOK257 cells on culture dishes was not affected by the expression of wild-type or mutant FLCN (Fig. 1C). However, anchorage independent growth measured as colony numbers on soft agar was low in the wildtype FLCN cell line UOK257-2, which expressed high levels of $F L C N$, compared to the parental UOK257 cell line
(UOK257-P) (Fig. 1D). One of the characteristics of the UOK257 cells was a slow growth rate (doubling time, $52+/-9$ hrs) on culture dishes. These cells also grew very slowly in soft agar taking 3-4 weeks to reach a countable colony size. By comparison, HT-1080 cells derived from a fibrosarcoma grew faster in soft agar and often generated larger colonies (data not shown).

\section{Tumor growth was suppressed by wild-type $F L C N$ but not by mutant $\mathrm{FLCN}-\mathrm{H} 255 \mathrm{R}$ expression}

To examine whether the tumorigenic potential of UOK257 cells was affected by wild-type or mutant FLCN, mutant FLCN (UOK257-P and -H255R) and wild-type $F L C N$-expressing cells (UOK257-2, $-3,-4$ and -6) were injected subcutaneously with matrigel into athymic nude mice and tumor growth was measured for up to one year. Most of the mice injected with UOK257-P and UOK257H255R cells developed solid tumors, although some animals only developed flat patches of tumor cells (See addi- 
A

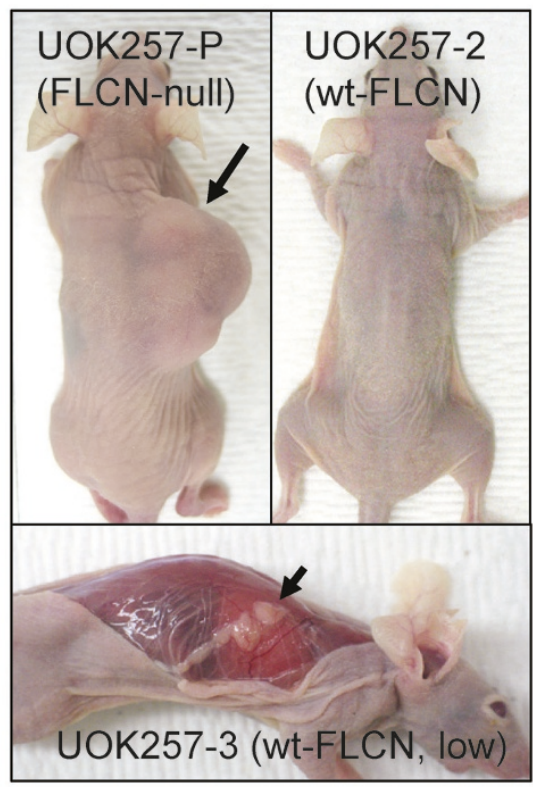

B

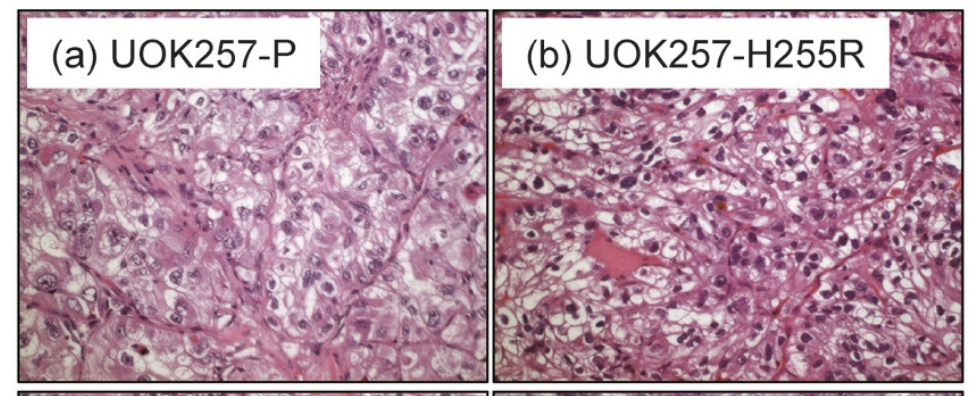

(c) UOK257-3

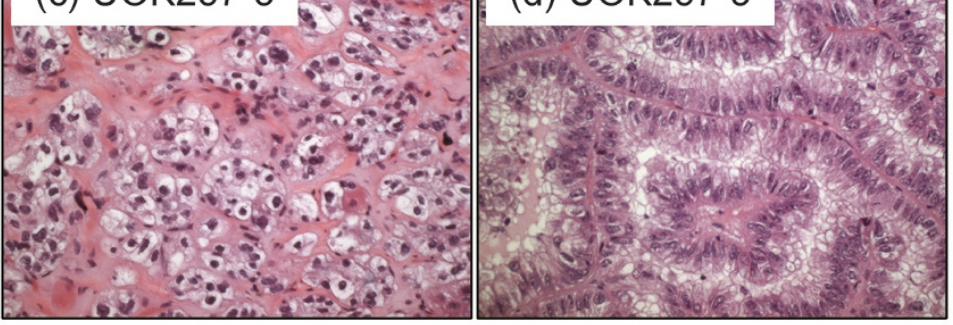

Figure 2 Suppression of tumorigenesis by re-introduction of wild-type $F L C N$ not mutant $F L C N$ in the $F L C N$-null cell line, UOK257. (A) Tumorigenesis of UOK257 cells in nude mice was suppressed by wild-type FLCN. Arrow indicates a tumor from UOK257-P cells. Arrowhead indicates a flat patch of tumor cells from UOK257-3 cells. (B) Histologies of the tumors from different clones of UOK257 cells. (a) high, (b) medium, and (c) low grade clear cell histologies; (d) papillary RCC.

tional file 1: Table S1 and Fig. 2A). All of these tumors were high grade and exhibited clear cell histology (See additional file 1: Table S1 and Fig. 2B). On the other hand, the mice injected with UOK257 cells expressing a high level of FLCN (UOK257-2, -4 and -6) did not develop tumors (See additional file 1: Table S1 and Fig. 2A). Instead, flat masses of cells only rarely containing tumor cells were observed in 6 of 35 (17\%) of the animals. The mice injected with UOK257-3 cells expressing a very low level of $F L C N$ developed solid tumors with low incidence $(2 / 10)$ and smaller size (See additional file 1: Table S1). In some animals, UOK257-3 cells grew as flat patches (5/10; Fig. 2A) and exhibited mostly clear cell histology with varying grades (low to high; Fig. 2B).

We investigated whether wild-type or mutant FLCN transgenes, or the endogenous mutant FLCN genes were lost during tumor progression. Genomic DNA was isolated from the tumors or tumor cell patches and PCR was performed using a primer pair specific to exon 10 and exon 11 that amplifies $664 \mathrm{bp}$ of the endogenous FLCN gene or $99 \mathrm{bp}$ of the $F L C N$ transgene. All of the tumors from the cell lines retained the endogenous mutant FLCN gene (c.1285dupC) and all of the tumors from UOK257-3 and UOK257-H255R retained their respective transgenes (See additional file 1: Fig. S1).
Gene expression microarray analysis identified genes regulated by $F L C N$ and the pathways in which they interact To identify the genes regulated by FLCN expression, we performed gene expression microarray analysis using RNAs isolated from the UOK257 cell lines expressing either no, mutant or wild-type FLCN. We identified a total of 439 genes, which were up or down-regulated more than 2-fold in the mutant and FLCN-null cell lines (UOK257-P and UOK257-H255R) compared to the wildtype FLCN cell lines (UOK257-2, -3, -4 and -6) (See additional file 2: Table S2). To explore the biological processes and pathways regulated by $F L C N$, the genes were subclassified with the help of "Panther Classification System" http://www.pantherdb.org and three prominent pathways were identified, namely cadherin signaling, TGF- $\beta$ signaling, and angiogenesis (Fig. 3A). Although all three of these pathways are important in tumorigenesis, we focused on the genes involved in TGF- $\beta$ signaling (See additional file 1: Fig. S2). We found that TGF- $\beta 2$ (TGFB2), Inhibin $\beta A$ (INHBA, a subunit of activin A), SMAD3 (SMAD3) and thrombospondin-1 (THBS1) were down-regulated, and Gremlin (GREM1) was upregulated in FLCN-null and mutant FLCN-H255R UOK257 cells compared with FLCN-restored UOK257 cells. We confirmed the GREM1, TGFB2, INHBA, SMAD3 and THBS1 

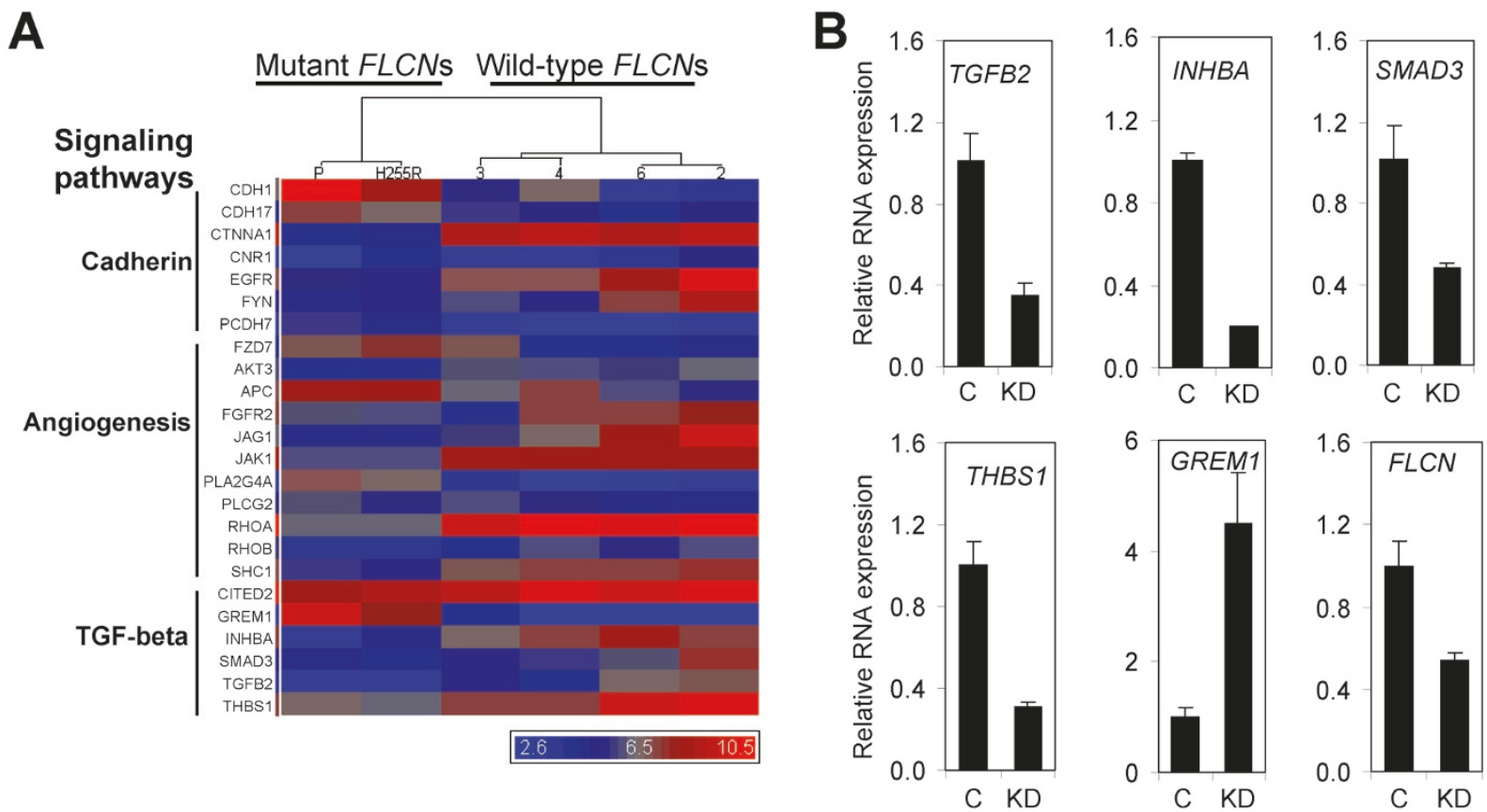

Figure 3 Genes differentially expressed in mutant and wild-type FLCN cell lines. (A) Heat-map representation of the genes differentially expressed in mutant and wild-type FLCN cell lines, and hierarchical clustering of the cell lines. Gene symbols are listed on the left side of each row. Three prominent groups of genes involved in signaling pathways were indicated on the left side of the gene symbols. (B) Deregulation of the key molecules in TGF- $\beta$ signaling by FLCN knockdown in the UOK257-2 cells expressing wildtype FLCN. C, control cell line infected with empty retrovirus; KD, FLCNknockdown cell line infected with a retrovirus expressing short hairpin RNA targeting FLCN.

microarray results by quantitative RT-PCR (See additional file 1: Fig. S3).

\section{Knockdown of FLCN deregulates TGFB2, INHBA, GREM1, THBS1 and SMAD3 expression in FLCN-restored UOK257 cells}

We next examined whether the expression levels of TGFB2, INHBA, THBS1, GREM1 and SMAD3 could be deregulated by knockdown of FLCN in FLCN-restored UOK 257 cells. A FLCN-knockdown cell line was generated by introducing a retrovirus that expressed shRNA against FLCN in FLCN-restored cells (UOK257-2). In addition to reduced expression of $F L C N$, the expression of TGFB2, INHBA, THBS1 and SMAD3 was decreased and the expression of GREM1 was increased in the FLCN-knockdown cell line (Fig. 3B).

GREM1, TGFB2, INHBA, THBS1 and SMAD3 expression levels were down-regulated in BHD-associated renal tumors

In order to determine whether the genes that were regulated by FLCN in in vitro cell culture were differentially expressed in renal tumors from BHD patients compared to normal kidney parenchyma, we performed quantitative RT-PCR using RNA isolated from these tissues. GREM1, TGFB2, INHBA, THBS1 and SMAD3 RNA expression levels were significantly lower in the BHD renal tumors compared to normal kidney tissue (Fig. 4A). However, FLCN RNA levels were not statistically different $(\mathrm{P}=0.316)$. In support of the RT-PCR data, immunohistochemical staining of TGF- $\beta 2$ showed strong TGF- $\beta 2$ expression in the normal renal tubules but reduced expression in the tumors from BHD patients (Fig. 4B, left panel). In addition, the UOK257 xenograft tumors expressed lower levels of TGFB2 compared to normal mouse kidney (Fig. 4B, right panel).

We measured protein expression of SMAD2, SMAD3, phospho-SMAD3 (pSMAD3) and FLCN in renal tumors from BHD patients $(\mathrm{n}=11)$ and normal human kidney tissue $(n=5)$. pSMAD3 levels were high in 3 out of 5 normal kidneys but only 1 (T11) of 11 tumors (Fig. 4C). In addition, SMAD3 levels and SMAD3/SMAD2 ratios were higher in normal kidneys compared to the tumors. On the other hand FLCN protein levels were lower or undetectable in all tumors except T11, in which a moderate level of FLCN expression was detected along with high levels of pSMAD3 and SMAD3 (Fig. 4C). Therefore it is likely that the T11 tumor was contaminated with normal kidney tissue.

In order to investigate whether receptor mediated TGF- $\beta$ signaling was disrupted by the loss of FLCN expression, TGF- $\beta$ induced SMAD3 phosphorylation was 
examined in UOK257 cells and compared to UOK257-2 cells. TGF- $\beta$ induced SMAD3 phosphorylation was not affected by FLCN inactivation (Fig. 5A). In addition, BMP4 induced SMAD1/5/8 phosphorylation was not disrupted by loss of FLCN expression (data not shown). We then examined whether TGF- $\beta$ induced gene expression was dysregulated in FLCN-null UOK257 cells. SMAD7, an inhibitory SMAD, is known to be induced by TGF- $\beta$. SMAD7 expression was induced in both UOK257 and UOK257-2 cell lines (Fig. 5B). However the basal and the maximal induced levels of SMAD7 were two fold greater in UOK257-2 cells than in UOK257 cells. Similar to SMAD7, TGFB2 and INHBA expressions were induced by TGF- $\beta$ in both cell lines but their basal and maximal levels of expression were substantially higher (5.8- to 23fold) in FLCN-restored UOK257-2 cells compared with FLCN-null UOK257 cells (Fig. 5B).

Since FLCN and FNIP1/2 can complex with AMPK, and phosphorylation of these proteins is affected by AMPK and mTOR signaling, we wanted to know whether AMPK and mTOR signaling affected the expression of $T G F B 2$ and $I N H B A$ in a $F L C N$-dependent manner. Interestingly, both TGFB2 and INHBA were induced by the AMPK activator AICAR but reduced by the AMPK inhibitor Compound $C$ in UOK257-2 cells expressing $F L C N$ as well as in cells in which FLCN expression was knocked down by a retrovirus expressing FLCN shRNA [UOK257-2/FLCN-KD; Fig. 5C(a) and Fig. 5C(b)]. Rapamycin, an inhibitor of mTOR signaling, also induced TGFB2 and INHBA expression in both FLCN-expressing UOK257-2 and UOK257-2/FLCN-KD cells [Fig. 5C(a) and Fig. $5 \mathrm{C}(\mathrm{b})]$. However, the basal and maximal levels of induction of TGFB2 and INHBA by AICAR and rapamycin were higher in FLCN-expressing UOK257-2 cells compared to UOK257-2/FLCN-KD cells [Fig. 5C(a) and Fig. $5 \mathrm{C}(\mathrm{b})$ ]. Similar results were obtained from an experiment using UOK257 FLCN-null and UOK257-2 FLCNexpressing cell lines. Interestingly, AICAR induced TGFB2 and INHBA expression in UOK257-2 cells but not in UOK257 cells [Fig. 5C(c) and Fig. 5C(d)].

\section{Activin A but not TGF- $\beta 2$ suppressed anchorage- independent growth of UOK257 cells}

Initially, in order to confirm that protein expression levels were consistent with the mRNA expression levels, we measured secreted TGF- $\beta 2$ and activin A levels in the media of UOK257 and UOK257-2 cells by ELISA. In accordance with their mRNA expression, TGF- $\beta 2$ and activin A protein expression levels were lower in UOK257 cells compared to UOK257-2 cells (Fig. 6A and 6B). Since both TGF- $\beta 2$ and activin A have been reported to suppress cell growth, we examined their effect on growth of UOK257 cells. To evaluate the growth suppressive effects of TGF- $\beta 2$ and activin A, UOK257 cells were treated with
TGF- $\beta 2$ or activin A and cultured for 4 weeks in soft-agar. Unexpectedly, TGF- $\beta 2$ appeared to increase colony formation of UOK 257 cells at both $1 \mathrm{ng} / \mathrm{ml}$ and $5 \mathrm{ng} / \mathrm{ml}$ (Fig. $6 \mathrm{C})$. However, activin A reduced colony formation at 1 $\mathrm{ng} / \mathrm{ml}$ and completely suppressed colony formation at 5 ng/ml (Fig. 6D).

\section{Discussion}

UOK257 is the only renal cancer cell line available to date that has been established from a BHD patient's tumor tissue. This cell line is particularly valuable for study of the biological role of $F L C N$ inactivation in tumorigenesis because it harbors a $F L C N$ mutation predicted to produce only truncated mutant protein and induces the growth of tumors in vivo with histology resembling the BHD-associated renal tumor from which it was derived [23]. In this study, we have established and characterized UOK257 cell lines in which wild-type or mutant $F L C N$ was stably expressed. Although anchorage dependent cell growth in vitro was not affected by wild-type $F L C N$ expression, cell growth in vivo and anchorage-independent growth in soft agar were severely diminished by the expression of wildtype $F L C N$. We have searched for downstream target genes regulated by FLCN through gene expression microarray analysis and identified a number of genes that were differentially expressed in wild-type $F L C N$ (UOK257-2, -4, and -6) compared with mutant FLCN and $F L C N$-null (UOK257-H255R and -P) cells. We found three prominent groups of genes involved in cadherin signaling, TGF- $\beta$ signaling, and angiogenesis. Notably, several key genes involved in TGF- $\beta$ signaling, such as TGFB2, INHBA, THBS1 and SMAD3, were down-regulated in $F L C N$-null and mutant $F L C N$ cells as well as in the BHD-associated renal tumors. Consistently, GREM1, the antagonist of BMP that signals through SMADs was highly up-regulated in mutant $F L C N$ and $F L C N$-null UOK257 cells although its expression was low in BHDassociated renal tumors.

We observed that the expression level of FLCN is important for tumor suppression, since the UOK257 cell lines (UOK257-4, -2 and -6) expressing high levels of FLCN did not develop tumors whereas the UOK257-3 cell line expressing a very low level of FLCN, did develop tumors with a low incidence ( 2 out of 10 ). It is likely that the FLCN expression level in UOK257-3 cells is marginal for tumor suppression, allowing tumor growth in some animals but suppressing tumor growth in others. In support of this idea, the expression levels of the downstream target genes in UOK257-3 cells were either similar to FLCN-null and FLCN mutant cells (UOK257-P and UOK257-H255R), or midway between the FLCN-null$F L C N$ mutant group and the $F L C N$-restored group, which expressed high levels of FLCN (UOK257-2 and UOK257-6) (See additional file 1: Fig. S3). 
A

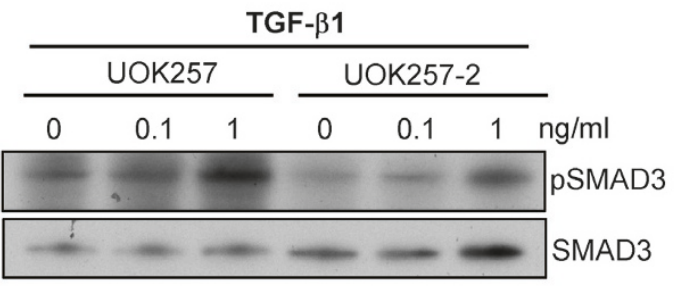

B
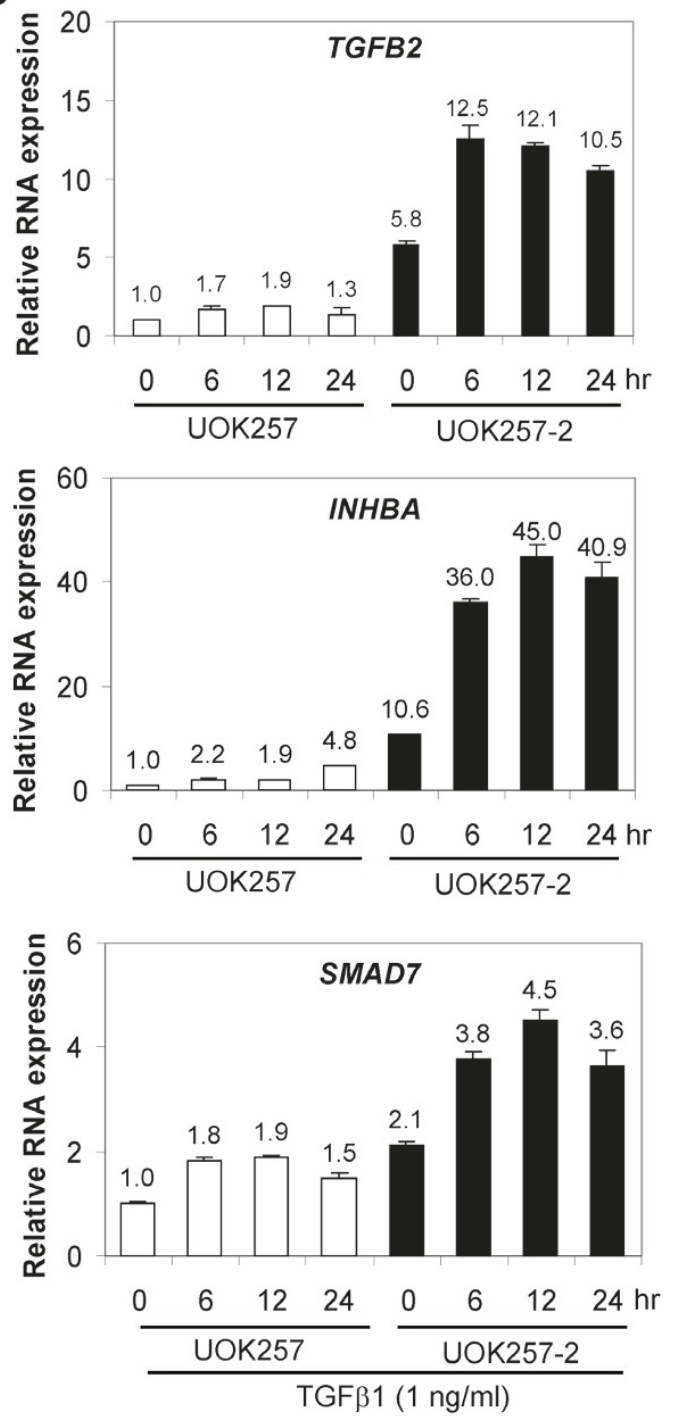

C
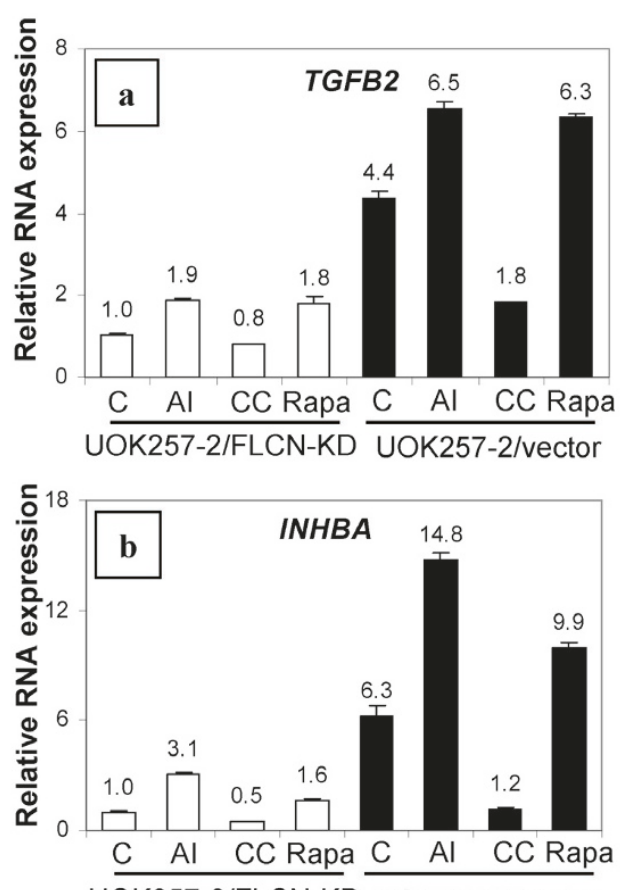

UOK257-2/FLCN-KD UOK257-2/vector
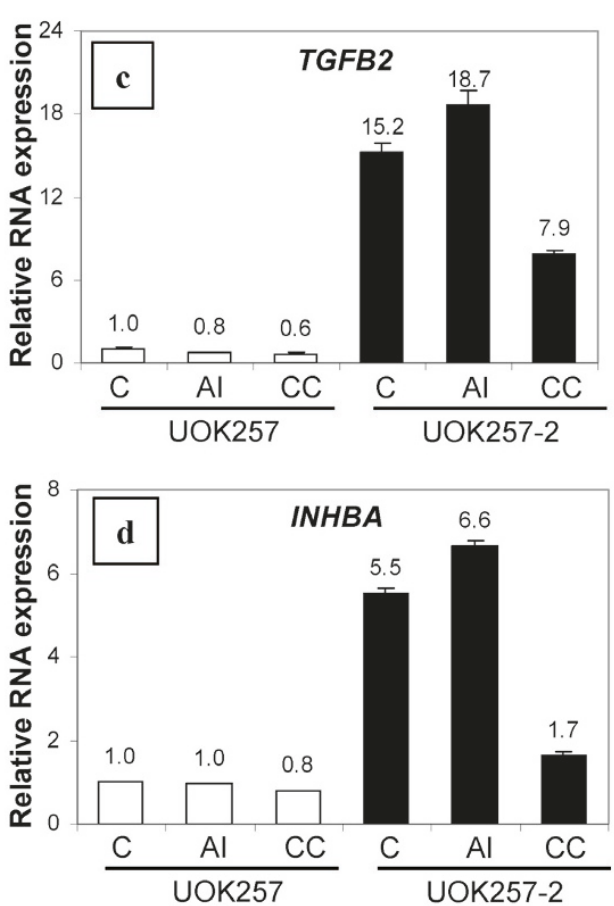

Figure 5 TGF- $\beta 1$ induced SMAD3 phosphorylation and effects of AICAR, Compound $C$ and rapamycin on TGF- $\beta 1$ induction of TGFB2 and INHBA in FLCN-null and FLCN-expressing cells. (A) TGF- $\beta 1$ induced SMAD3 phosphorylation in UOK257 and UOK257-2 cells. Cells were treated with $0,0.1$ and $1 \mathrm{ng} / \mathrm{ml}$ of TGF- $\beta 1$ for $1 \mathrm{hr}$. (B) TGF- $\beta 1$ induced TGFB2, INHBA and SMAD7 mRNA expression in UOK257 and UOK257-2 cells. Cell were treated with $1 \mathrm{ng} / \mathrm{ml}$ of TGF- $\beta 1$ for $0,6,12$ and $24 \mathrm{hr}$ after serum starvation for $12 \mathrm{hr}$. (C) (a and b) Cells were serum-starved for $24 \mathrm{hr}$ and treated with $0.5 \mathrm{mM}$ AICAR (Al), $20 \mu \mathrm{M}$ Compound C (CC) or $1 \mathrm{nM}$ rapamycin (Rapa) for $12 \mathrm{hr}$. UOK257-2/vector, UOK257-2 cells infected with empty retrovirus; UOK257-2/ FLCN-KD, UOK257-2 cells infected with retrovirus expressing shRNA targeting FLCN. (c and d) Cells were serum-starved for 15 hr and treated with 0.5 mM AICAR or $20 \mu \mathrm{M}$ Compound C (CC) for $6 \mathrm{hr}$. 


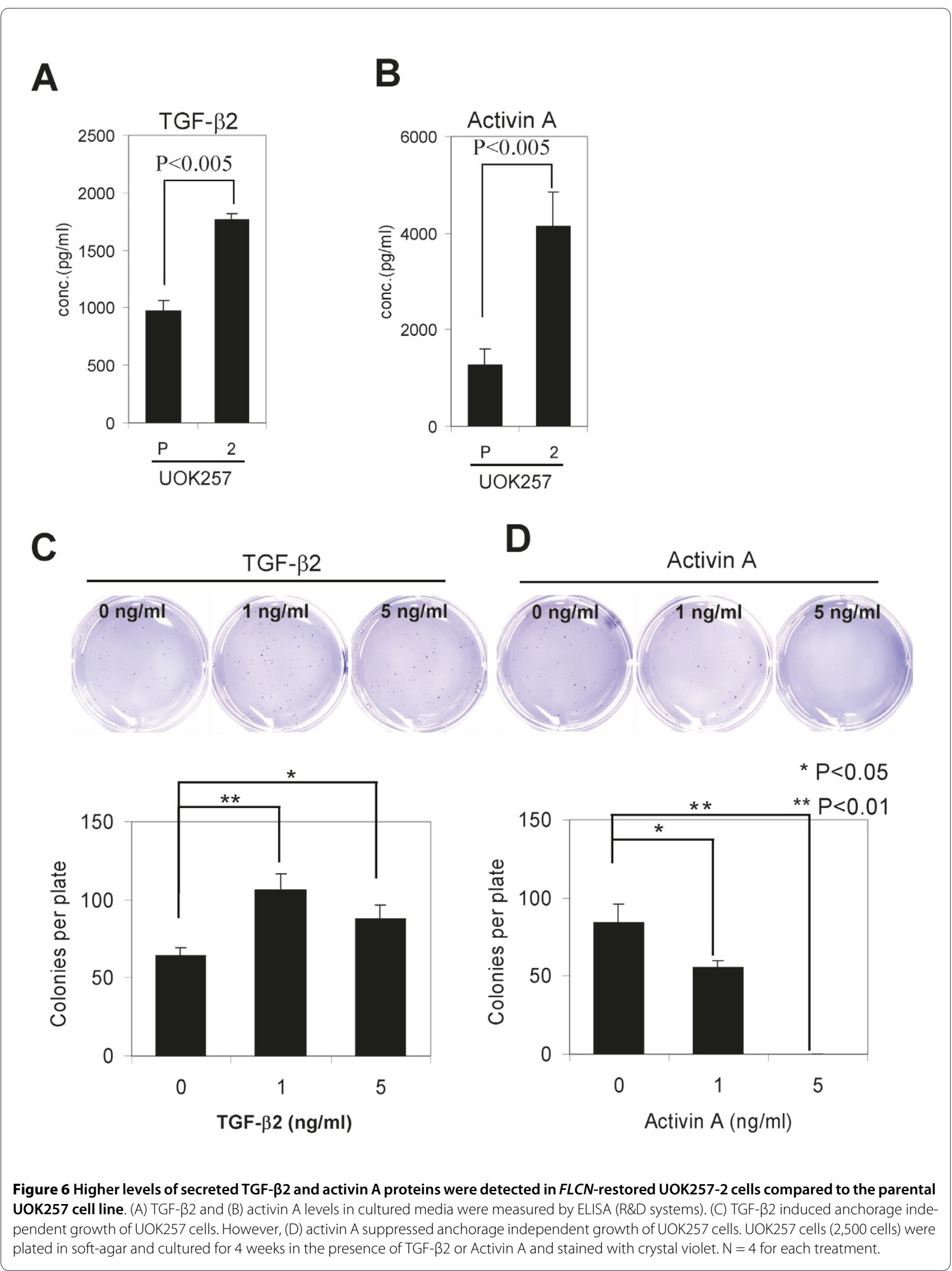


UOK257-H255R cells expressed a low level of FLCN protein resulting in loss of tumor suppressor function and deregulation of TGF- $\beta$ signaling, even though they expressed slightly more FLCN mRNA than UOK257-4 cells (Fig.1A and 1B). These data suggest that FLCNH255R missense mutant protein found in the canine model of BHD syndrome is less stable than wild-type FLCN. Thus decreased stability of mutant FLCN is likely to contribute to the loss of FLCN tumor suppressor function.

It has been suggested that Drosophila BHD ( $d B H D)$ regulates germline stem cell (GSC) maintenance downstream or in parallel with Jak/Stat and dpp (BMP ortholog in Drosophila) signaling [24]. $d B H D$ knockdown by siRNA suppressed overproliferation of GSC induced by hyperactivation of Jak/Stat or dpp signaling. Interestingly, Jak1, encoding a kinase that transmits signals by phosphorylating Stats in cells, was identified by microarray analysis as a downregulated gene in the mutant FLCN and FLCN-null cells (Fig. 3A). We also identified several key genes in TGF- $\beta$ /BMP signaling such as TGFB2, $I N H B A, T H B S 1$ and SMAD3 (a regulatory SMAD) that were down-regulated in the mutant FLCN and FLCN-null cells. On the other hand, GREM1, which encodes a protein that binds and inactivates BMP activity, was upregulated in the mutant and FLCN-null cells. Thus the genetic interactions between $d B H D$, and Jak/Stat and dpp (BMP) signaling may be partially explained by FLCN deregulation of genes involved in these pathways.

The human TGF- $\beta$ superfamily consists of 42 members including TGF- $\beta \mathrm{s}$, activins, bone morphogenic proteins (BMPs), and growth and differentiating factors (GDFs) $[25,26]$. TGF- $\beta$ s are multi-functional cytokines that modulate cell proliferation, apoptosis, differentiation, adhesion and migration. TGF- $\beta$ shows a biphasic effect on tumor cell growth [27]. It inhibits tumor cell growth in the early phase of tumorigenesis but promotes cell growth when cells escape the anti-proliferative effect of TGF- $\beta$ in the late phase of tumorigenesis. Interestingly, TGF- $\beta 2$ induced anchorage independent growth of UOK257 cells (Fig. 6C), suggesting that UOK257 cells are refractory to the growth suppressive effect of TGF- $\beta$. The possibility exists that reduced expression of TGF- $\beta 2$ in FLCN-null cells contributed to cell growth in the early phase of tumorigenesis.

Disruption of TGF- $\beta$ signaling has been reported in many cancers. TGF- $\beta$ type II receptor is often mutated in gastro-intestinal cancers [28-30]. Mutations in SMAD2 or SMAD4 occur frequently in pancreatic and colorectal carcinomas [31-33]. Although mutations in SMAD3 have not been reported, 3 out of $8(37.5 \%)$ gastric tumors in one report showed low to undetectable levels of SMAD3 expression and restoration of SMAD3 suppressed tumorigenicity of gastric cancer cells [34]. Low levels of
SMAD3 expression in the BHD tumors may contribute to the ability of these renal tumor cells to escape the growth suppressive effect of TGF- $\beta$.

Activins are homo- or heterodimeric proteins consisting of two $\beta$ subunits ( $\beta A$ and $\beta B$ ), while inhibins are heterodimers of $\alpha$ and $\beta$ subunits (inhibin- $A[\alpha \beta A]$ and inhibin-B $[\alpha \beta B]$ ) [35]. INHBA is one of the $\beta$ subunits $(\beta \mathrm{A})$ that comprise activin $\mathrm{A}(\beta \mathrm{A} \beta \mathrm{A})$, activin $\mathrm{AB}(\beta \mathrm{A} \beta \mathrm{B})$ and inhibin $A(\alpha \beta A)$. Activin A regulates kidney organogenesis, tubular regeneration and renal fibrosis [reviewed in [36]]. Activins also induce apoptosis, and inhibit cell proliferation and tumor growth in numerous types of cells. In contrast to TGF- $\beta 2$, activin A inhibited growth of UOK257 cells in soft-agar (Fig. 6D), suggesting that activin signaling is intact in UOK257 cells. Thus reduced expression of INHBA, $\beta$ subunit of activin A, in UOK257 cells and BHD tumors, may be permissive for tumor cell growth. It would be interesting to examine whether activin A treatment can suppress BHD tumor growth in vivo.

Thrombospondin-1 (THBS1) is one of the five members of a family of thrombospondins that mediate the interaction of normal and cancer cells with the extracellular matrix and surrounding tissue. THBS1 suppresses tumor growth by activating TGF- $\beta$ and by inhibiting angiogenesis. THBS1 exerts direct effects on endothelial cell migration and survival through interaction with CD36. It also reduces availability of VEGF by inhibiting MMP9, therefore releasing VEGF from the extracellular matrix. There are several reports suggesting that reduced expression of THBS1 or hypermethylation of THBS1 is associated with poor prognosis of cancer patients and higher tumor grade [37-40]. Accordingly THBS1 regulation may be an important part of the tumor suppressor function of FLCN.

We examined whether TGF- $\beta$ signaling is dysregulated by the inactivation of the FLCN gene. TGF- $\beta$ or BMP4 induced SMAD3 or SMAD1/5/8 phosphorylation was not affected by FLCN inactivation suggesting receptor mediated SMAD phosphorylation is not altered by FLCN. However, several genes whose expressions are regulated by TGF- $\beta$ were dysregulated by the inactivation of FLCN. The basal and maximal induced levels of the downstream target genes (TGFB2, INHBA and SMAD7) regulated by TGF- $\beta$ were reduced in cells with FLCN inactivation. These data suggest that FLCN may regulate TGF- $\beta$ signaling through a non-SMAD mediated mechanism. As a result of such regulation, the level of TGF- $\beta$ ligands, such as TGF- $\beta 2$ and activin A, could be highly induced in cells expressing FLCN by a positive feedback control.

A possible function of FLCN in energy sensing and metabolism has been suggested by its interaction with AMPK through FNIP1/2 and by the observation that 
FLCN phosphorylation is affected by mTOR signaling (20-21). Here we demonstrated that an AMPK activator, AICAR, and an AMPK inhibitor, Compound $\mathrm{C}$, as well as an mTOR inhibitor, rapamycin, affected the expression of the same key molecules involved in TGF- $\beta$ signaling, which appear to be regulated by FLCN. Thus FLCN could be a key molecule connecting energy-sensing signals to growth suppressive TGF- $\beta$ signaling.

\section{Conclusions}

Here for the first time we have confirmed the tumor suppressor function of FLCN in vivo and identified new potential FLCN downstream targets in the TGF- $\beta$ signaling pathway. This study will provide a foundation for understanding the pathogenesis of BHD syndrome at the molecular level and be useful for finding therapeutic targets for treating BHD-associated kidney cancer and potentially, sporadic chromophobe RCC. We are currently analyzing the mechanism by which FLCN regulates these target genes and the functional importance of deregulation of these FLCN target genes in tumorigenesis.

\section{Methods}

\section{Establishment of cell lines, cell culture, and cell growth}

Wild-type or mutant (H255R) FLCN cDNA was transduced into UOK257 cells using the ViraPower Lentiviral expression system (Invitrogen) following the manufacturer's protocols. Stable clones were selected using Blasticidin $\mathrm{S}(1.5 \mu \mathrm{g} / \mathrm{ml})$. Cells were maintained in DMEM medium supplemented with $10 \%$ fetal bovine serum (FBS) and penicillin/streptomycin. To evaluate growth rate in culture, cells $\left(2 \times 10^{3}\right)$ were plated in each well of five 96 well plates, cultured, and cell numbers were measured at day 1, 2, 3, 5 and 7 using the CyQuant Cell Proliferation Assay Kit (Molecular Probes). Adenoviral vectors (pAd/CMV/V5-DEST) expressing wild-type and mutant (c.1285dupC) FLCN were generated using the ViraPower Adenoviral Gateway system (Invitrogen) following the manufacturer's protocol. A retroviral shRNA vector targeting $F L C N$ was generated by inserting double stranded oligonucleotides (forward sequence, 5'-GATCCCC GGTGTTTGAGGCAGAGCAGTTCAAGAGACTGCTCTG CCTCAAACACCTTTTTA-3' and reverse sequence, 5'GCTTAAAAAGGTGTTTGAGGCAGAGCAGTCTCTTGAACTGCTCTGCCTCAAACACCGGG-3') into HindIII and BglII sites of pSuper-Retro vector (Oligoengine) following the manufacturer's instruction. UOK257-2 cells were infected with the FLCN shRNA vectors and selected against puromycin $(7.5 \mathrm{ug} / \mathrm{ml})$.

\section{Colony formation assay}

UOK257 cells $\left(5 \times 10^{3}\right)$ were suspended in $1.5 \mathrm{ml}$ of $0.3 \%$ agar in DMEM containing 10\% FBS and were overlayed on $1.5 \mathrm{ml}$ of pre-solidified $0.5 \%$ agar in the same medium.
Cells were cultured in a $\mathrm{CO}_{2}$ incubator for 3-4 weeks. Colonies were stained for 1 hour with $0.02 \%$ crystal violet solution dissolved in $10 \%$ neutral formalin. Colony number was counted under a dissection microscope after washing with PBS three times.

\section{Tumor growth in nude mice}

Cells $\left(1 \times 10^{6}\right)$ suspended with basement membrane matrix (BD Biosciences) were injected subcutaneously into the flanks of athymic nude mice. Tumor growth was measured once a week and mouse health was monitored daily. Mice bearing tumors larger than $2 \mathrm{~cm}$, or showing severe health problems, were sacrificed and examined. Otherwise tumor growth was monitored for up to one year after injection. Tumors were fixed in 10\% buffered formalin solution for histological examination and flash frozen in liquid nitrogen for protein and RNA extraction. Animal care procedures followed NCI-Frederick Animal Care and Use Committee guidelines.

\section{Immunoblotting}

Cells were harvested and lysed in RIPA buffer $(50 \mathrm{mM}$ Tris-Cl, pH 8.0 with $150 \mathrm{mM} \mathrm{NaCl}, 1.0 \% \mathrm{NP}-40$ and $0.5 \%$ sodium deoxycholate) or $1 \times$ SDS sample buffer (Biorad). Cell lysates were resolved by 4-20\% SDS PAGE and blotted onto PVDF membrane. The following antibodies were used in this study: anti-FLCN mouse monoclonal [20], anti- $\beta$-actin (Sigma), anti-SMAD2/3 (Santa Cruz, sc-6032), and anti-pSMAD2/3 (Santa Cruz, sc-11769) antibodies. Immunoblots were processed by the ECL Detection System (Pierce) according to the manufacturer's protocols.

\section{Immunohistochemistry}

Paraffin tissue sections were deparaffinized, rehydrated in graded alcohol and boiled in Tris-EDTA buffer $\mathrm{pH} 8.0$ for $20 \mathrm{~min}$ at $90^{\circ} \mathrm{C}$ for antigen retrieval. After blocking, sections were probed with primary antibodies overnight and then incubated with HRP-polymer conjugated secondary antibodies. Diaminobenzidine hydrochloride (DAB) was used as a substrate for peroxidase. Sections were then briefly counterstained with hematoxylin and permanently mounted for observation.

\section{ELISA}

Cells $\left(2 \times 10^{5}\right)$ were cultured on 6 well plates for 3 days and culture media was collected for assay. TGF- $\beta 2$, and activin A levels in the media were quantified by Human TGF- $\beta 2$ DuoSet (R\&D systems) and activin A DuoSet (R\&D systems), respectively, following the manufacturer's instruction.

\section{RNA isolation, microarray analysis and pathway analysis}

Total RNAs were isolated from the UOK257 cell lines using Trizol reagent (Invitrogen) and further purified using RNeasy mini kit (QIAGEN) according to the manu- 
facturer's protocols. Probes, which were generated using these RNAs, were hybridized to the Human Genome U133 Plus 2.0 arrays (Affymetrix) and processed according to recommended protocols. The CEL files were processed using the Partek Genomic Suite 6.2 (Partek Inc.). Data were transformed using a log normalization process and the differentially expressed genes were identified by Student's t-test and Mann-Whitney U-test. The genes that were differentially expressed in mutant FLCN cell lines (UOK257-P and -H255R) and wild-type FLCN cell lines (UOK257-2, -4 and -6) were used for further analysis.

\section{Quantitative real-time reverse transcription-PCR (RT-PCR)}

To confirm the microarray results, quantitative real-time reverse transcription PCR (RT-PCR) was performed. RNAs were digested with DNase I for $30 \mathrm{~min}$ at $37^{\circ} \mathrm{C}$ followed by heat denaturation at $70^{\circ} \mathrm{C}$ for $20 \mathrm{~min}$ to remove genomic DNA contamination. Total RNAs $(2.5 \mu \mathrm{g})$ were primed with $100 \mathrm{ng}$ random primers and reverse-transcribed by Superscript II reverse transcriptase (Invitrogen) at $42^{\circ} \mathrm{C}$ for $1 \mathrm{hr}$. The identical reactions were performed without reverse transcriptase to generate negative controls. PCR primers were generated using Primer 3 software [41] or Primer Express 3.0 (Applied Biosystems). Quantitative RT-PCR was performed with Power SYBR-Green or Taqman Gene Expression Master Mix (Applied Biosystems) using a 7300 Real-Time PCR system (Applied Biosystems) following the manufacturer's protocols. All reactions were run in triplicate using $\beta$-actin, GAPDH or cyclophilin A genes as internal controls. The relative level of a particular gene expression was evaluated according to the function of $2^{-\mathrm{ddCt}}$, where $\mathrm{ddCt}$ is $\mathrm{dCt}_{\text {(treatment) }}-\mathrm{dCt}_{\text {(control) }}$, $\mathrm{dCt}$ is $\mathrm{Ct}_{\text {(target gene) }}-\mathrm{Ct}_{\text {(GAPDH or }}$ actin) and $\mathrm{Ct}$ is the cycle at which the threshold is crossed. The gene-specific primer pairs for the PCR reactions are as follows: FLCN forward 5'-TTCACGCCATTCCTACACCAGA-3' and reverse 5'-GCCCACAGGTTGTCATCACTTG-3', GREM1 forward 5'-GCAAAACCCAGC CGCTTAA-3' and reverse 5'-TGATGGTGCGACTGTTGCA-3', TGFB2 forward 5'-CGAGAGGAGCGACGAAGAGT-3' and reverse 5'-AGGGCGGCATGTCTA TTTTG-3', THBS1 forward 5'-CCAGATCAGGCAGACACAGA-3' and reverse 5'-AGTTGTCCCGTTCATTGAGG-3', INHBA forward 5'-TGGAGTGTGATG GCAAGGTCA-3' and reverse 5'-GCATGATAGCCAGAGGGAGCA-3', SMAD3 forward 5'-GACGAGGTCTGCGTGAATCC-3' and reverse 5'-GTGGCGTGGCACC AACA-3', and GAPDH forward 5'-TTCCACCCATGGCAAATTCC-3' and reverse 5'-CGCCCCACTTGATTTTGGAG-3'. SMAD7 forward 5'-CCAACTGC AGACTGTCCAGA-3' and reverse 5'-CAGGCTCCAGAAGAAGTTGG-3'. PCR product quality was monitored using post-PCR dissociation curve analysis.

\section{Additional material}

Additional file $\mathbf{1}$ table S1, and figures S1, S2, S3. Table S1. Frequency and characteristics of tumors that developed from different UOK257 cell lines in athymic nude mice. Figure S1. PCR amplification of endogenous FLCN (endo) and FLCN transgene (tg) from the xenograft tumors. Figure S2. Deregulation of the key molecules in TGF- $\beta$ signaling by FLCN expression. Figure S3. Quantitative RT-PCR for TGFB2, INHBA, THBS1, GREM1 and SMAD3 in the UOK257 cell lines expressing either mutant or wild-type FLCN.

Additional file $\mathbf{2}$ Table S2. Table S2. The genes differentially expressed in the wild-type FLCN and mutant FLCN cell lines.

\section{Competing interests}

The authors declare that they have no competing interests.

\section{Authors' contributions}

$\mathrm{SBH}$ designed the experiments. SBH, VAV, HBO, JS, DTN and MB performed the experimental work. MB generated critical cell lines for the work. MJM performed histopathologic analysis. SBH wrote the manuscript. WML and LSS contributed to the design of the experiments, review of the data, scientific discussions and manuscript editing. All authors read and approved the final manuscript.

\section{Acknowledgements}

We thank the Laboratory of Molecular Technology of SAIC-Frederick, Inc. for performing microarray hybridizations and generation of data files and Louise Cromwell for excellent animal technical support. This research was supported in part by the Intramural Research Program of the NIH, National Cancer Institute, Center for Cancer Research. This project has been funded in part with federal funds from the National Cancer Institute, National Institutes of Health, under contract HHSN261200800001E. The content of this publication does not necessarily reflect the views or policies of the Department of Heath and Human Services, nor does mention of trade names, commercial products, or organizations imply endorsement by the U.S. Government. NCI-Frederick is accredited by AAALAC International and follows the Public Health Service Policy for the Care and Use of Laboratory Animals. Animal care was provided in accordance with the procedures outlined in the "Guide for Care and Use of Laboratory Animals (National Research Council; 1996; National Academy Press; Washington DC).

\section{Author Details}

1 Urologic Oncology Branch, Center for Cancer Research, National Cancer Institute, 10 Center Drive MSC1107, 10/CRC/1W-5940, Bethesda, MD 20892 USA, ${ }^{2}$ Laboratory of Pathology, Center for Cancer Research, National Cancer Institute, 10 Center Drive MSC1516, 10/MCC/2B50, Bethesda, MD 20892 USA and ${ }^{3}$ Basic Science Program, SAIC-Frederick, Inc, NCI-Frederick, Frederick, MD 21702 USA

Received: 19 January 2010 Accepted: 23 June 2010 Published: 23 June 2010

\section{References}

1. Birt AR, Hogg GR, Dube WJ: Hereditary multiple fibrofolliculomas with trichodiscomas and acrochordons. Arch Dermatol 1977, 113:1674-1677.

2. Toro JR, Glenn G, Duray P, Darling T, Weirich G, Zbar B, Linehan M, Turner ML: Birt-Hogg-Dube syndrome: a novel marker of kidney neoplasia. Arch Dermatol 1999, 135:1195-1202.

3. Zbar B, Alvord WG, Glenn G, Turner M, Pavlovich CP, Schmidt L, Walther M, Choyke P, Weirich G, Hewitt SM, Duray P, Gabril F, Greenberg C, Merino MJ, Toro J, Linehan WM: Risk of renal and colonic neoplasms and spontaneous pneumothorax in the Birt-Hogg-Dube syndrome. Cancer Epidemiol Biomarkers Prev 2002, 11:393-400.

4. Schmidt LS, Nickerson ML, Warren MB, Glenn GM, Toro JR, Merino MJ, Turner ML, Choyke PL, Sharma N, Peterson J, Morrison P, Maher ER, Walther MM, Zbar B, Linehan WM: Germline BHD-mutation spectrum and phenotype analysis of a large cohort of families with Birt-HoggDube syndrome. Am J Hum Genet 2005, 76:1023-1033.

5. Toro JR, Wei MH, Glenn G, Weinreich M, Toure O, Vocke C, Turner M, Choyke P, Merino MJ, Pinto PA, Steinberg SM, Schmidt LS, Linehan WM: $B H D$ mutations, clinical and molecular genetic investigations of Birt- 
Hogg-Dube Syndrome: A new series of 50 families and a review of published reports. J Med Genet 2008, 45:321-331.

6. Pavlovich CP, Walther MM, Eyler RA, Hewitt SM, Zbar B, Linehan WM, Merino MJ: Renal tumors in the Birt-Hogg-Dube syndrome. Am J Surg Pathol 2002, 26:1542-1552.

7. Khoo SK, Bradley M, Wong FK, Hedblad MA, Nordenskjold M, Teh BT: BirtHogg-Dube syndrome: mapping of a novel hereditary neoplasia gene to chromosome 17p12-q11.2. Oncogene 2001, 20:5239-5242.

8. Schmidt LS, Warren MB, Nickerson ML, Weirich G, Matrosova V, Toro JR, Turner ML, Duray P, Merino M, Hewitt S, Pavlovich CP, Glenn G, Greenberg $C R$, Linehan WM, Zbar B: Birt-Hogg-Dube syndrome, a genodermatosis associated with spontaneous pneumothorax and kidney neoplasia maps to chromosome 17p11.2. Am J Hum Genet 2001, 69:876-882.

9. Nickerson ML, Warren MB, Toro JR, Matrosova V, Glenn G, Turner ML, Duray P, Merino M, Choyke P, Pavlovich CP, Sharma N, Walther M, Munroe D, Hill R, Maher E, Greenberg C, Lerman MI, Linehan WM, Zbar B, Schmidt LS: Mutations in a novel gene lead to kidney tumors, lung wall defects, and benign tumors of the hair follicle in patients with the Birt-HoggDube syndrome. Cancer Cell 2002, 2:157-164.

10. Khoo SK, Giraud S, Kahnoski K, Chen J, Motorna O, Nickolov R, Binet O, Lambert D, Friedel J, Lévy R, Ferlicot S, Wolkenstein P, Hammel P, Bergerheim U, Hedblad MA, Bradley M, Teh BT, Nordenskjöld M, Richard S: Clinical and genetic studies of Birt-Hogg-Dube syndrome. J Med Genet 2002, 39:906-912.

11. Leter EM, Koopmans AK, Gille JJ, van OSTA, Vittoz GG, David EF, Jaspars EH, Postmus PE, van Moorselaar RJ, Craanen ME, Starink TM, Menko FH: BirtHogg-Dube syndrome: clinical and genetic studies of 20 families. $J$ Invest Dermatol 2008, 128:45-49.

12. Vocke CD, Yang Y, Pavlovich CP, Schmidt LS, Nickerson ML, Torres-Cabala CA, Merino MJ, Walther MM, Zbar B, Linehan WM: High frequency of somatic frameshift $B H D$ gene mutations in Birt-Hogg-Dube-associated renal tumors. J Natl Cancer Inst 2005, 97:931-935.

13. Okimoto K, Sakurai J, Kobayashi T, Mitani H, Hirayama Y, Nickerson ML, Warren MB, Zbar B, Schmidt LS, Hino O: A germ-line insertion in the BirtHogg-Dube $(B H D)$ gene gives rise to the Nihon rat model of inherited renal cancer. Proc Natl Acad Sci USA 2004, 101:2023-2027.

14. Lingaas F, Comstock KE, Kirkness EF, Sorensen A, Aarskaug T, Hitte C, Nickerson ML, Moe L, Schmidt LS, Thomas R, Breen M, Galibert F, Zbar B, Ostrander EA: A mutation in the canine BHD gene is associated with hereditary multifocal renal cystadenocarcinoma and nodular dermatofibrosis in the German Shepherd dog. Hum Mol Genet 2003, 12:3043-3053

15. Baba M, Furihata M, Hong SB, Tessarollo L, Haines DC, Southon E, Patel V, Igarashi P, Alvord WG, Leighty R, Yao M, Bernardo M, lleva L, Choyke P, Warren MB, Zbar B, Linehan WM, Schmidt LS: Kidney-targeted Birt-HoggDube gene inactivation in a mouse model: Erk1/2 and Akt-mTOR activation, cell hyperproliferation, and polycystic kidneys. J Natl Cancer Inst 2008, 100:140-154.

16. Chen J, Futami K, Petillo D, Peng J, Wang P, Knol J, Li Y, Khoo SK, Huang D, Qian CN, Zhao P, Dykema K, Zhang R, Cao B, Yang XJ, Furge K, Williams BO, Teh BT: Deficiency of FLCN in mouse kidney led to development of polycystic kidneys and renal neoplasia. PLoS One 2008, 3:e3581

17. Hasumi Y, Baba M, Ajima R, Hasumi H, Valera VA, Klein ME, Haines DC, Merino MJ, Hong SB, Yamaguchi TP, Schmidt LS, Linehan WM: Homozygous loss of BHD causes early embryonic lethality and kidney tumor development with activation of mTORC1 and mTORC2. Proc Natl Acad Sci USA 2009, 106:18722-18727.

18. Hartman TR, Nicolas E, Klein-Szanto A, Al Saleem T, Cash TP, Simon MC, Henske EP: The role of the Birt-Hogg-Dube protein in mTOR activation and renal tumorigenesis. Oncogene 2009, 28:1594-1604.

19. Hudon V, Sabourin S, Dydensborg AB, Kottis V, Ghazi A, Paquet M, Crosby K, Pomerleau V, Uetani N, Pause A: Renal tumor suppressor function of the Birt-Hogg-Dube syndrome gene product folliculin. J Med Genet 2010, 47:182-189.

20. Baba M, Hong SB, Sharma N, Warren MB, Nickerson ML, Iwamatsu A, Esposito D, Gillette WK, Hopkins RF, Hartley JL, Furihata M, Oishi S, Zhen W, Burke TR Jr, Linehan WM, Schmidt LS, Zbar B: Folliculin encoded by the BHD gene interacts with a binding protein, FNIP1, and AMPK, and is involved in AMPK and mTOR signaling. Proc Natl Acad Sci USA 2006, 103:15552-15557.

21. Hasumi H, Baba M, Hong SB, Hasumi Y, Huang Y, Yao M, Valera VA, Linehan WM, Schmidt LS: Identification and characterization of a novel folliculin-interacting protein FNIP2. Gene 2008, 415:60-67.
22. van Slegtenhorst M, Khabibullin D, Hartman TR, Nicolas E, Kruger WD, Henske EP: The Birt-Hogg-Dube and tuberous sclerosis complex homologs have opposing roles in amino acid homeostasis in Schizosaccharomyces pombe. J Biol Chem 2007, 282:24583-24590.

23. Yang Y, Padilla-Nash HM, Vira MA, Abu-Asab MS, Val D, Worrell R, Tsokos M, Merino MJ, Pavlovich CP, Ried T, Linehan WM, Vocke CD: The UOK 257 cell line: a novel model for studies of the human Birt-Hogg-Dube gene pathway. Cancer Genet Cytogenet 2008, 180:100-109.

24. Singh SR, Zhen W, Zheng Z, Wang H, Oh SW, Liu W, Zbar B, Schmidt LS, Hou SX: The Drosophila homolog of the human tumor suppressor gene $B H D$ interacts with the JAK-STAT and Dpp signaling pathways in regulating male germline stem cell maintenance. Oncogene 2006 , 25:5933-5941

25. Derynck R, Feng XH: TGF-beta receptor signaling. Biochim Biophys Acta 1999, 1333:F105-150.

26. Shi Y, Massague J: Mechanisms of TGF-beta signaling from cell membrane to the nucleus. Cell 2003, 113:685-700.

27. de Caestecker MP, Piek E, Roberts AB: Role of transforming growth factor-beta signaling in cancer. J Nat/ Cancer Inst 2000, 92:1388-1402.

28. Markowitz S, Wang J, Myeroff L, Parsons R, Sin L, Lutterbaugh J: Inactivation of the type II TGF-beta receptor in colon cancer cells with microsatellite instability. Science 1995, 268:1336-1338.

29. Parsons R, Myeroff LL, Liu B, Willson JK, Markowitz SD, Kinzler KW, Vogelstein B: Microsatellite instability and mutations of the transforming growth factor beta type II receptor gene in colorectal cancer. Cancer Res 1995, 55:5548-5550.

30. Lu SL, Akiyama Y, Nagasaki H, Saitoh K, Yuasa Y: Mutations of the transforming growth factor-beta type II receptor gene and genomic instability in hereditary nonpolyposis colorectal cancer. Biochem Biophys Res Commun 1995, 216:452-457.

31. Hahn SA, Schutte M, Hoque AT, Moskaluk CA, da Costa LT, Rozenblum E, Weinstein CL, Fischer A, Yeo CJ, Hruban RH, Kern SE: DPC4, a candidate tumor suppressor gene at human chromosome 18q21.1. Science 1996, 271:350-353

32. MacGrogan D, Pegram M, Slamon D, Bookstein R: Comparative mutational analysis of DPC4 (Smad4) in prostatic and colorectal carcinomas. Oncogene 1997, 15:1111-1114.

33. Han SU, Kim HT, Seong DH: Loss of the Smad3 expression increases susceptibility to tumorigenicity in human gastric cancer. Oncogene 2004, 23:1333-1341

34. Takagi Y, Kohmura H, Futamura M, Kida H, Tanemura H, Shimokawa K, Saji $\mathrm{S}$ : Somatic alterations of the DPC4 gene in human colorectal cancers in vivo. Gastroenterology 1996, 111:1369-1372.

35. Chen YG, Wang Q, Lin SL, Chang CD, Chuang J, Ying SY: Activin signaling and its role in regulation of cell proliferation, apoptosis, and carcinogenesis. Exp Biol Med (Maywood.) 2006, 231:534-544.

36. Maeshima A, Miya M, Mishima K, Yamashita S, Kojima I, Nojima Y: Activin A: autocrine regulator of kidney development and repair. Endocr $J$ 2008, 55:1-9.

37. loachim E, Michael MC, Salmas M, Damala K, Tsanou E, Michael MM, Malamou-Mitsi V, Stavropoulos NE: Thrombospondin-1 expression in urothelial carcinoma: prognostic significance and association with p53 alterations, tumour angiogenesis and extracellular matrix components. BMC Cancer 2006, 6:140.

38. Sutton CD, O'Byrne K, Goddard JC, Marshall LJ, Jones L, Garcea G, Dennison AR, Poston G, Lloyd DM, Berry DP: Expression of thrombospondin-1 in resected colorectal liver metastases predicts poor prognosis. Clin Cancer Res 2005, 11:6567-6573.

39. Rice AJ, Steward MA, Quinn CM: Thrombospondin 1 protein expression relates to good prognostic indices in ductal carcinoma in situ of the breast. J Clin Pathol 2002, 55:921-925.

40. Guerrero D, Guarch R, Ojer A, Casas JM, Ropero S, Mancha A, Pesce C, Lloveras B, Garcia-Bragado F, Puras A: Hypermethylation of the thrombospondin-1 gene is associated with poor prognosis in penile squamous cell carcinoma. BJU Int 2008, 102:747-755.

41. Rozen $\mathrm{S}$, Skaletsky H: Primer3 on the www for general users and for biologist programmers. Methods Mol Biol 2000, 132:365-386.

doi: $10.1186 / 1476-4598-9-160$

Cite this article as: Hong et al., Tumor suppressor FLCN inhibits tumorigenesis of a FLCN-null renal cancer cell line and regulates expression of key molecules in TGF-? signaling Molecular Cancer 2010, 9:160 